\title{
Study on Dynamic load characteristics of circular arc tooth synchronous belt based on RecurDyn
}

\author{
Jianhua Guo ${ }^{1, \mathrm{a}}$, Qingxin Meng ${ }^{1, \mathrm{~b}}$, Xin $\mathrm{Yu}^{1, \mathrm{c}}$, Qingming $\mathrm{Hu}^{1, \mathrm{~d}}$ \\ ${ }^{1}$ Qiqihar University College of Electrical Engineering, 161006,Qiqihar, China \\ a guo1034@ sina.com, ${ }^{\mathrm{b}}$ mengqingxin890831@163.com, ${ }^{\mathrm{c}} 18745292065 @ 163 . c o m,{ }^{\mathrm{d}}$ huqingming1267@126.com
}

\begin{abstract}
Using the UG software, the new type arc tooth synchronous belt and pulley solid model are created. In view of the synchronous belt and pulley transmission belongs to the multi rigid multi flexible coupled hybrid multi body system dynamics problem, carry out the flexible treatment of synchronous belt by HyperMesh, and import into the multi body dynamics software RecurDyn and the dynamic virtual prototype simulation model of the new type arc tooth synchronous belt gear transmission is built, simulation analysis about the load distribution of the dedendum and addendum of teeth in transmission system is made under different gap, reveal the rule of effects on stress distribution of synchronous belt analyzed in detail under different gap. The results showed that the stress of the belt tooth increases together with increasing of the side gap on the transmission process. A digital simulation and design method to design the new type arc tooth synchronous belt tooth shape, analysis of the transmission performance are provided.
\end{abstract}

Keywords-synchronous belt;drive;FEM;MFBD;Recurdyn

\section{INTRODUCTION}

Dynamic load distribution of Synchronous belt is an important factor that influences the life. Kagotani [1-3] and others research the transmission error of oblique toothed synchronous belt and synchronous belt transmission errors in initial tension when resonance under the action by experiment in the quasi static conditions. Domek [4] established a single tooth synchronous belt static coupling model to study the problem of the synchronous belt and pulley meshing contact stress. Koyama [5] study the leap characteristics of trapezoidal tooth synchronous belt based on the finite element method and experimental. Milanovic [6] analysis the impact of torque on the load distribution of synchronous belt based on finite element.

But the research model has limitations, can not reflect the dynamic performance of synchronous belt. So this paper aims to the synchronous belt flexibility based on continuous method, establishing dynamics equation of dynamic contact coupling belt drive system; realize the true meaning of rigid flexible coupled analysis, effects the load distribution of backlash on the belt tooth.

\section{THE KINETIC EQUATION OF DYNAMIC CONTACT COUPLING BELT DRIVE SYSTEM}

In this paper, the rigid belt continuous flexible for the representation of flexible belt to an infinite number of degrees of freedom of a plurality of unit limited nodal degrees of freedom, these nodes of the element elastic deformation can be represented approximately by linear combination of a small amount of modal [7-10].

Flex the rigid belt for a certain number of grid units (Fig .1), connect with node between each grid unit, $r_{k-1}$ and $r_{k}$ respectively the position vector of the body unit nodes $k-1$ and $k$ of the flexible belt in the inertial coordinate system. The coordinate system $x_{k-1}^{\prime} y_{k-1}^{\prime} z_{k-1}^{\prime}$ of node $k-1$ for the inscribed coordinate of node $k$, $x_{(k-1) k}^{\prime} y_{(k-1) k}^{\prime} z_{(k-1) k}^{\prime}$ is the coordinate system for nodes connected. The origin is located at the node $k$ of corresponding the second bit serial number in subscript and the direction of the flexible belt parallel to the inscribed coordinate system $x_{k-1}^{\prime} y_{k-1}^{\prime} z_{k-1}^{\prime}$ of the node $k$. Describe the deformation of belt (caused by tension, bending and other factors) by node force and act on the nodes connecting origin coordinate system $x_{(k-1) k}^{\prime} y_{(k-1) k}^{\prime} z_{(k-1) k}^{\prime}$. Select the node coordinate $q_{(k-1) k}^{f}$ of belt unit as generalized coordinates of flexible flat belt, said the relative coordinate of node $k$ related with the internal coordinates system $x_{k-1}^{\prime} y_{k-1}^{\prime} z_{k-1}^{\prime}$.

Assembled the nodal force of all body elements and obtain the generalized force of elastic and damping force of flexible belt material by coordinate transformation. Therefore the absolute nodal displacement of the flexible belt recursive superimposed by the relative node displacement of body element.

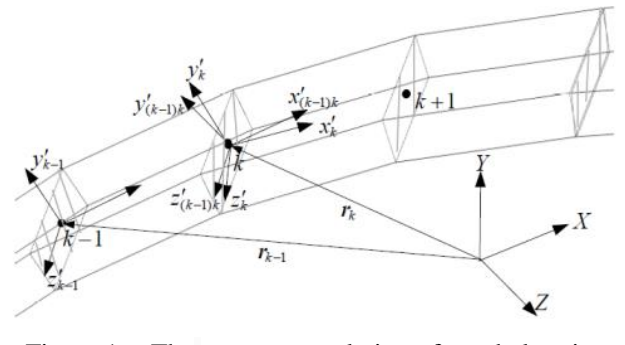

Figure 1. The recurrence relation of two belt units

A. The dynamic contact model of flexible belt and pulley

Set up the dynamic contact model of belt body element with rigid pulley by the hysteresis contact dynamics method of permeation of contact body relative to the target. Use the relative relationship of the surface contact 
to surface between belt body unit and pulley to predict the contact state and the contact force calculation. The belt body unit as a contact body and pulley as objects, the contact surface of the belt body unit are described with contact point and the contact surface of pulley with a certain number of grid unit, as shown in Fig .2. Therefore, the displacement of the contact point of the belt body unit relative to the target surface of the pulley

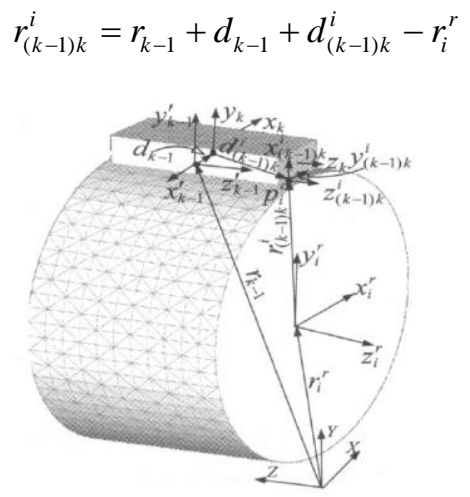

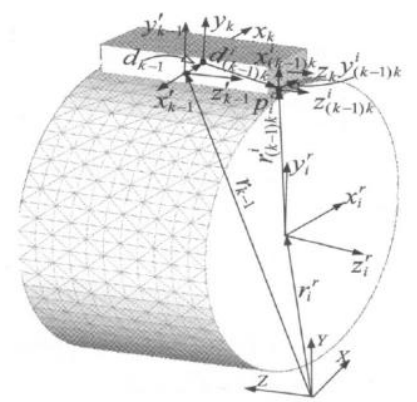

Figure 2. Relationship of belt body unit contact with driving pulley

The infiltration capacity of belt body unit relative to the pulley $i$ at the contact point $p$

$$
\delta_{p}^{i}=\left(r_{(k-1) k}^{i}\right)_{y_{i}^{r}}-R_{i}^{r}
$$

Type $R_{i}^{r}$ is the effective radius of driving pulley $i$, $\left(r_{(k-1) k}^{i}\right)_{y_{i}^{r}}$ is the projection component of the relative displacement in direction of the driving pulley body coordinate $y_{i}^{r}$, which contains the moving coordinate of the pulley $\theta_{i}$ (angle of pulley).

By Kuhn-Tucker contact determination conditions known: (a) $\delta_{p}^{i} \geq 0$, non contact; (b) $\left(F_{p}^{i}\right)_{n}<0$ contact pressure;(c) $\left(F_{p}^{i}\right)_{n} \delta_{p}^{i}=0$, no penetration and the contact force $0 ;(\mathrm{d})\left(F_{p}^{i}\right)_{n} \dot{\delta}_{p}^{i}=0$, the continuous contact force. The nonlinear hysteresis normal contact force of the flat belt and the driving pulley

$$
\begin{aligned}
\left(F_{p}^{i}\right)_{n}= & K_{c}\left(\delta_{p}^{i}\right)^{m 1}+\operatorname{step}\left(\delta_{p}^{i}, 0,0, \delta_{\max }, c_{0}\right) \times \\
& \operatorname{sign}\left(\dot{\delta_{p}^{i}}\right) \times\left|\dot{\delta}_{p}^{i}\right|^{m 2} \times\left(\delta_{p}^{i}\right)^{m 3}
\end{aligned}
$$

Type $\operatorname{step}\left(\delta_{p}^{i}, 0,0, \delta_{\max }, c_{0}\right)$ is the haversine step function, $K_{c}$ is the normal contact stiffness, $\delta_{p}^{i}$ and $\dot{\delta}_{p}^{i}$ as the normal permeability and normal relative velocity of the belt body unit and driving pulley at the contact point $\mathrm{p}, \delta_{\max }$ is the maximum penetration of the contact damping of the contact pair in $c_{\max }=c_{0}$. The non-linear relationship of the normal contact force and contact penetration volume is determined by $m 1$; the non-linear relationship of the contact damping force and normal relative velocity is determined by damping exponent $m 2$; the damping effect index $m 3 \quad(m 3 \geqslant 1)$ can effectively solve the negative numerical problem of the contact force due to infiltration volume too small.

Determine the stick slip friction characteristics of the belt and driving pulley by Coulomb friction law. In the dynamic contact process, the friction coefficient and the relative velocity relationship of contact point and the nonlinear friction force are:

$$
\begin{gathered}
\mu_{p}^{i}\left(v_{p}^{i}\right)=\left\{\begin{array}{l}
\operatorname{step}\left(v_{p}^{i}, 0,0, v_{s}, \mu_{s}\right) \operatorname{sign}\left(-v_{p}^{i}\right) \\
v_{p}^{i} \leq\left|v_{s}\right| \\
\operatorname{step}\left(v_{p}^{i}, v_{s}, \mu_{s}, v_{d}, \mu_{d}\right) \operatorname{sign}\left(-v_{p}^{i}\right) \\
\left|v_{s}\right| \leq v_{p}^{i} \leq\left|v_{d}\right| \\
\mu_{d}, v_{p}^{i} \geq\left|v_{d}\right|
\end{array}\right. \\
\left(F_{p}^{i}\right)_{\tau}=-u_{p}^{i}\left(v_{\tau}\right)\left(F_{p}^{i}\right)_{n} \bullet\left(v_{\tau} /\left|v_{\tau}\right|\right)
\end{gathered}
$$

Type $v_{s}, v_{d}, \mu_{s}$ and $\mu_{d}$ are the relative static critical speed, relative critical dynamic speed, static friction coefficient and the dynamic friction coefficient at the contact point of the belt with the driving pulley, $v_{p}^{i}$ is the relative velocity at the contact point $p,\left(F_{p}^{i}\right)_{\tau}$ and $v_{\tau}$ are respectively tangential friction force and tangential relative velocity.

The force at the contact point $p$

$$
\left(F_{p}^{i}\right)_{c}=\left(F_{p}^{i}\right)_{n} n_{p}^{i}+\left(F_{p}^{i}\right)_{\tau} \tau_{p}^{i}
$$

Type $n_{p}^{i}$ and $\tau_{p}^{i}$ are the normal and tangential vector at the contact point $p$, calculate the force and moment components of the corresponding coordinate system by type.

B. The multi body contact coupling dynamic equation of the flexible belt drive system

As shown in Fig .3, choose the generalized coordinates $q=\left\{\begin{array}{lll}\theta_{1} & \theta_{2} & \theta_{3}\end{array}\right\}^{T}$, where the coordinate of node $k$ are $q_{(k-1) k}^{f}$ and $q^{f}=\left[\begin{array}{lll}q_{01}^{f} & q_{12}^{f} \ldots q_{(k-1) k}^{f}\end{array}\right]$ with respect to the nodes inscribed coordinate system $x_{k-1}^{\prime} y_{k-1}^{\prime} z_{k-1}^{\prime}$.

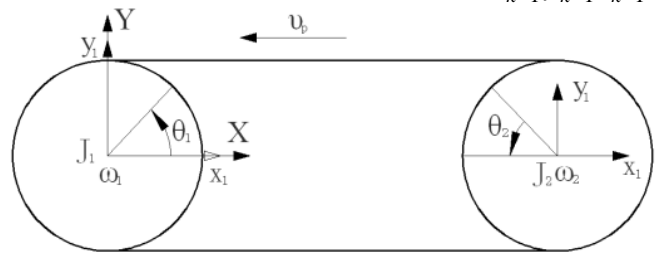

Figure 3. The model of belt drive system with two pulleys

Dynamic equations of the drive are obtained by Lagrange method. 


$$
J_{i} \Theta_{i}^{i}=Q_{F}^{i}-Q_{f}^{c i}(\mathrm{i}=1,2)
$$

The dynamics equation of flexible belt

$$
M^{b e}=Q_{c e}-Q_{f}^{b e}
$$

Type $J_{i}, Q_{F}^{i}$ respectively the moment of inertia and load of the pulley $i, Q_{f}^{c i}$ is the contact loads between flexible belt and pulley i. $Q_{c e}=T_{0}+m^{\prime} v_{b}^{2} / g$ is the initial tension and centrifugal force of the flexible belt, $v_{b}$ is the average line speed of the flexible belt, $Q_{f}^{b e}=Q_{f}^{c b e}+Q_{f}^{c i}$, $Q_{f}^{c b e}$ is the contact loads between flexible belt and pulley $i$, $\mathrm{g}$ is the acceleration of gravity.

$$
Q_{f}^{c k}=F_{e}^{c k}+F_{d}^{c k}+F_{\tau}^{c k}=G(p, \& t), k \text { respectively the }
$$
pulley $i$ or the flexible belt $b e$, where $F_{e}^{c k}, F_{d}^{c k}$ and $F_{\tau}^{c k}$ respectively the elastic contact force, damping force and friction force. $Q_{f}^{\prime}=F_{e}+F_{d}$ is material load of belt, where $F_{e}$ and $F_{d}$ are respectively the material elastic force and damping force of belt. Introduce the constraints of pulley, the matrix form of type (7) and (8)

$$
\left[\begin{array}{ccc}
J_{1} & & 0 \\
& J_{1} & \\
0 & & M_{n \times n}^{b e}
\end{array}\right]\left(\begin{array}{c}
Q_{1} \\
Q_{2}^{2} \\
()_{n \times 1}
\end{array}\right)=\left(\begin{array}{c}
Q_{F}^{1} \\
Q_{F}^{2} \\
Q_{c e}
\end{array}\right)-\left(\begin{array}{l}
Q_{f}^{c 1} \\
Q_{f}^{c 2} \\
Q_{f}^{b e}
\end{array}\right)-\Phi_{p}^{T} \lambda
$$

Type $J_{i}, Q_{F}^{i}$ respectively for the moment of inertia and load of the pulley $i, Q_{f}^{c i}$ is the contact load of the flexible belt and pulley $i, Q_{c e}$ is the initial tension of the flexible belt. $\Phi_{p}^{T}, \lambda$ and $Q_{F}^{i}$ respectively for the constraint equations and the Lagrange multiplier of the belt drive system. Put the constraint equation $\Phi_{p}^{T}$ into system by the way of the binding manner. Calculations the dynamic contact force of belt and pulley and solve the dynamic equations of the system.

\section{THE PROCESS OF FINITE ELEMENT ANALYSIS}

As shown in Fig .4 for the synchronous belt and belt pulley model of finite element simulation. The number of the belt tooth is 60 , the length of the pitch line is $571.5 \mathrm{~mm}$, the wide of the belt is $24 \mathrm{~mm}$, the number of the pulley teeth is 22 , and the pitch diameter is $66.702 \mathrm{~mm}$.

As shown in Fig .5, the synchronous belt strength layer is expressed by 2- node beam elements. The rubber of belt backing is ignored because the transmission force is hardly transmitted by this part. The rubber belt is an R type tooth. The tooth rubber and the bag cloth layer are expressed by the quadrilateral plane strain elements (number of elements: 27801). Table I shows the band properties of the material used in this analysis. All the materials are isotropic.

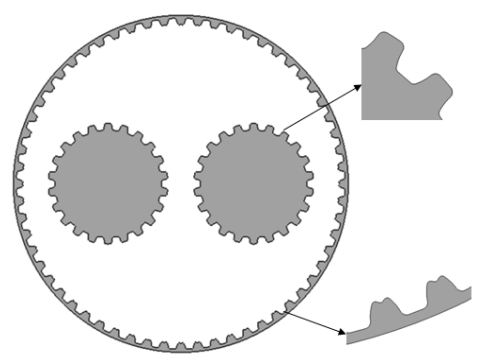

Figure 4. 3D model of synchronous belt and pulley

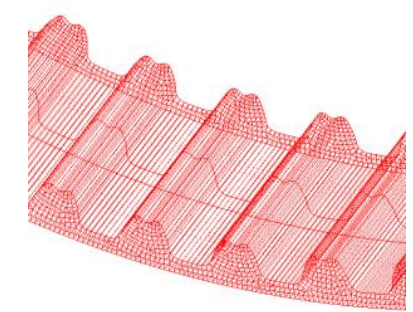

Figure 5. The mesh local model of synchronous belt

TABLE I. MATERIAL PROPERTIES OF SYNCHRONOUS BELT OF EACH LAYER

\begin{tabular}{|c|c|c|c|}
\hline & $\begin{array}{c}\text { Chloroprene } \\
\text { rubber }\end{array}$ & Glass fiber & Nylon cloth \\
\hline $\begin{array}{c}\text { Density } \\
{\left[\mathrm{kg} / \mathbf{m}^{3}\right]}\end{array}$ & $1.27 \times 10^{3}$ & 2.6 & 1.15 \\
\hline $\begin{array}{c}\text { Modulus of } \\
\text { elasticity } \\
{[\mathbf{M P a}]}\end{array}$ & $0.045 \times 10^{3}$ & 0.094 & 0.042 \\
\hline Poisson's ratio & $0.46 \times 10^{3}$ & 0.4 & 0.43 \\
\hline
\end{tabular}

Fig .6 shows the virtual prototype model. The center of the driving pulley is fixed and the driven pulley is shifted to the right side until the shaft load reaches $400 \mathrm{~N}$. Next, the driving pulley is made to rotate clockwise, until the speed reaches $1000 \mathrm{r} / \mathrm{min}$ by the step function and the torque is gradually increased until reaches $4500 \mathrm{~N} \cdot \mathrm{mm}$ in the driven pulley at the same time.

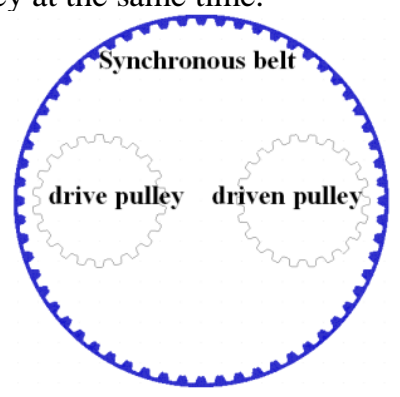

(a) Initial condition

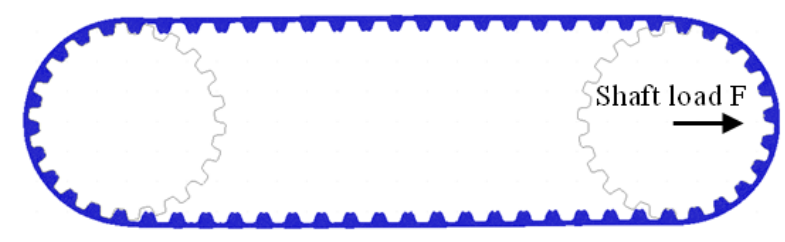

(b) Applied shaft load for driven pulley 


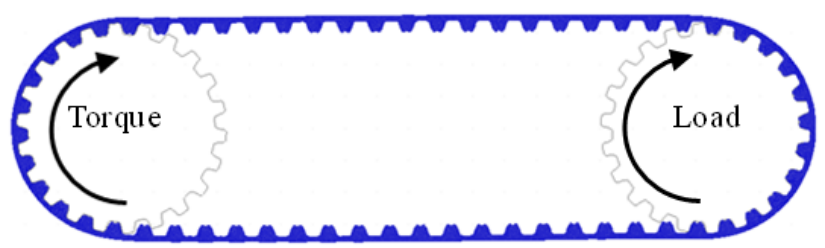

(c) Applied torque and load

Figure 6. The model of virtual prototype

\section{THE SIMULATION RESULTS AND ANALYSIS}

The initial shaft load is equal to $400 \mathrm{~N}$ in the belt, simulation time is $0.3 \mathrm{~s}$ and the step is 100 . Fig .5 shows the meshing conditions and the stress distributions of belt teeth.

As shown in Fig .7(a), the strength layer of belt tight side and slack side load. The belt tooth load decreases gradually together with the belt tooth meshing to pulley groove and the belt tooth load increases gradually together with the belt tooth out of meshing to pulley groove in the process of belt meshing to pulley. Fig .7(b) and (c) show the process of driving pulley and belt into and out of meshing. While into and out of meshing, have meshing interference for pitch difference at the first and the last tooth with pulley. When the belt tooth meshing into the pulley, belt tooth load decreases together with the pulley rotate, and when the belt tooth out of meshing the pulley groove, belt tooth and pulley groove have interference, made the stress of the belt tooth increases.

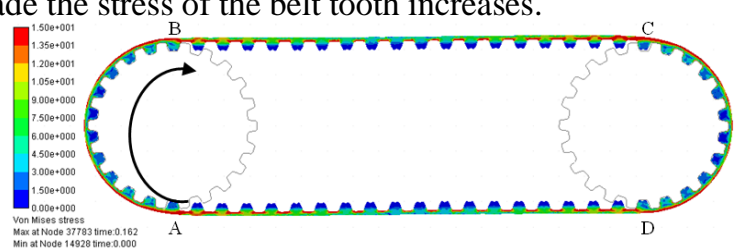

(a) Stress distribution of synchronous belt system

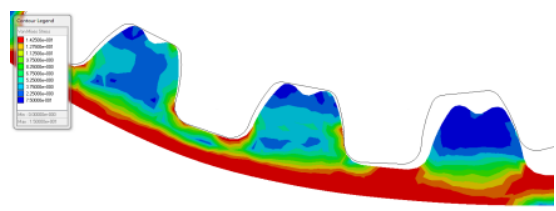

(b) Stress distribution of belt teeth at A

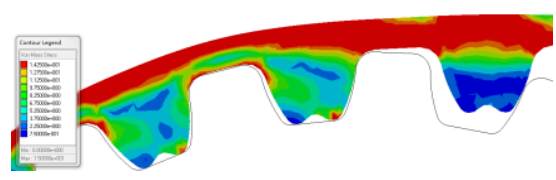

(c) Stress distribution of belt teeth at B

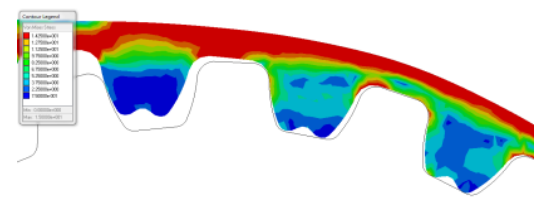

(d) Stress distribution of belt teeth at $\mathrm{C}$

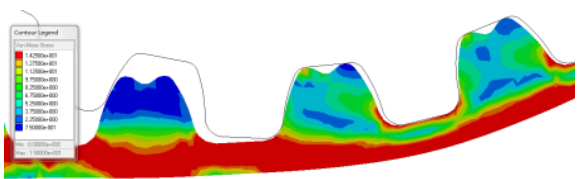

(e) Stress distribution of belt teeth at D

Figure 7. Stress distribution of synchronous belt

Fig .7 (d) and (e) show the load distribution of belt in the process of driven pulley and belt into and out of meshing. The change trend of driven pulley consistent with the driving pulley. The results of simulation close to the numerical modeling results of Koyama.

In order to analyze the load distribution of the tooth with the same simulation method in side gap respectively provided for the $0.1 \mathrm{~mm}, 0.2 \mathrm{~mm}, 0.3 \mathrm{~mm}$ and other settings parameters remain unchanged in simulation. Fig .8(a) study the position of belt tooth. The stress change curve under different side gap as shows in Fig .8(b-d) and measures the maximum stress at point $\mathrm{A}$. The maximum stress values were $38.2 \mathrm{~N}, 43.5 \mathrm{~N}$ and $49.8 \mathrm{~N}$ under side gap respectively $0.1 \mathrm{~mm}, 0.2 \mathrm{~mm}$ and $0.3 \mathrm{~mm}$. At the same time that can directly reflect the stress at the tooth root $\mathrm{C}$ is slightly less than A, the value of the stress at the tooth top $\mathrm{B}$ is far less than the root in the side gap of $0.2 \mathrm{~mm}$ and $0.3 \mathrm{~mm}$. The difference of the stress is larger in both sides root and the force in tooth top larger while in $0.1 \mathrm{~mm}$.

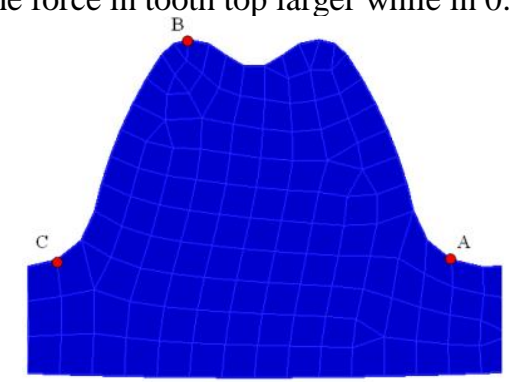

(a) Dedendum and addendum of belt tooth using analysis

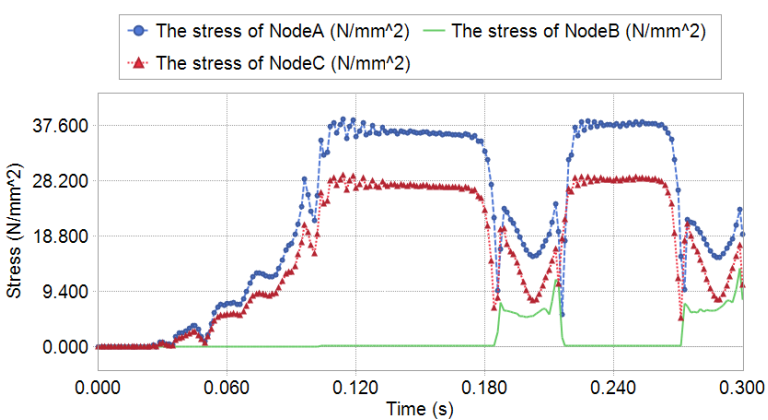

(b) The stress of belt tooth under side gap $0.1 \mathrm{~mm}$ 


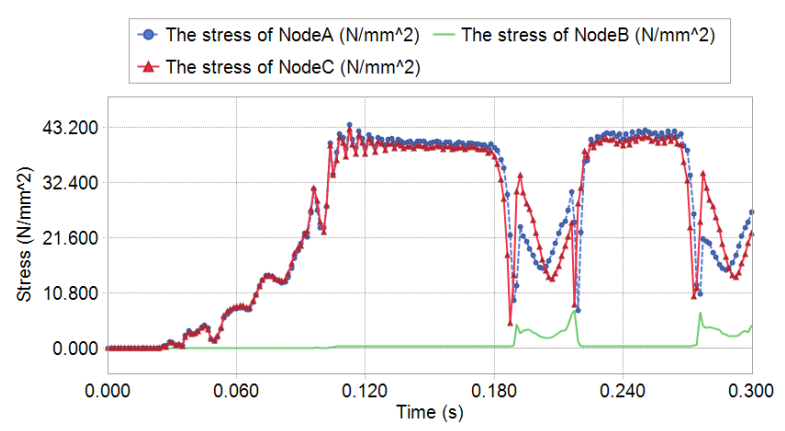

(c) The stress of belt tooth under side gap $0.2 \mathrm{~mm}$

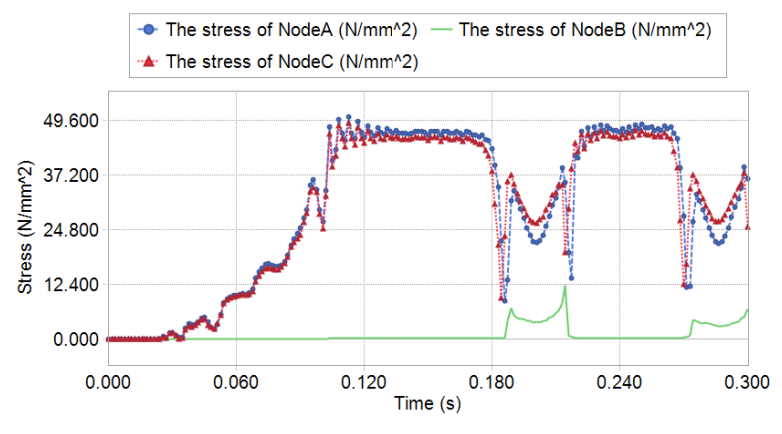

(d) The stress of belt tooth under side gap $0.3 \mathrm{~mm}$

Figure 8. The stress of belt tooth under different side gap

This is because the side gap is small and extrusion mutually in teeth, so pulley generated force to the belt tooth and the other side of the belt tooth contact with the adjacent pulley while belt meshing with pulley. Therefore, the difference of the value of stress between the belt tooth on both sides. When the side gap is large, belt tooth is noncontact with adjacent pulley in impact, so the value of stress of belt teeth root on both sides is not. The study can be seen the belt tensile stress increases together with increasing of side gap in the process of synchronous belt transmission, The change trend of the stress distribution on both sides of belt tooth root is agreement, but the amplitude of variation is quite different, the change trend of the stress in top keep with each position of the tooth.

\section{CONCLUSIONS}

Three dimensional model of synchronous belt transmission is established by UG software, and the belt flexible treatment by the software HyperMesh of CAE. Dynamic analysis on the transmission process of synchronous belt based on rigid flexible coupling technology and virtual prototype technology, draws the following conclusion:

(1) In the process of belt meshing to pulley, the belt tooth load decreases gradually together with the belt tooth meshing to pulley groove, the belt tooth load increases gradually together with the belt tooth out of meshing to pulley groove. The results of simulation and the numerical modeling analysis of Koyama are similar and the dynamic characteristics of belt more practical.

(2) That can be seen through the analysis on the transmission process of synchronous belt, the belt tooth stress increases together with increasing of the side gap. The change trend of the stress distribution on both sides of belt tooth root is agreement, but the amplitude of variation is quite different and the change trend of the stress in top keep with each position of the tooth.

\section{ACKNOWLEDGMENT}

This project is supported by National Science Foundation of China (51175273); Science and technology project of Qiqihar (GYGG-201313); the innovation of scientific research of Qiqihar University graduate (YJSCX2014-024X).

\section{REFERENCES}

[1] M. Kagotani, H. Ueda, T. Koyama. "Transmission Error in Helical Timing Belt Drives(Case of a Period of Pulley Pitch)," ASME Journal of Mechanical Design. Vol.123, No.3, Mar.2011, pp.104 110,doi: 10.1115/1.1326916.

[2] M. Kagotani, H. Ueda. "Transmission Error in Synchronous Belt With Resonance Under Installation Tension," Journal of Mechanical Design, vol.134, No.6, Jun.2012, pp.061003-1-12, doi: 10.1115/1.4006524

[3] K. Masanori, U. Hiroyuki. "Influence of Installation Tension on Transmission Error Due to Resonance in a Synchronous Belt,' Proceedings of the ASME 2014 12th Biennial Conference on Engineering Systems Design and Analysis, 2014, pp.1-10,doi: ESDA2014-20049.

[4] G. Domek. "Research on the Contact Area between the Timing Belt and the Toothed Pulley," Proceedings of the World Congress on Engineering, vol III, Jul.2011,pp.2242-2244,ISSN: 2078-0966.

[5] T. Koyama, Weiming Zhang, et al. "A Study on Jumping Characteristics in Synchronous Belt Drives(Experimental Results and FE analysis at driven pulley jumping)," ASME 2003 Design Engineering Technical Conferences and Computers and Information in Engineering Conference(DETC2003/PTG)," 2003 pp,1-6(CD-R), DETC2003 PTG-48009.

[6] Ivan MILANOVI Ć, Blaža STOJANOVIĆ. "Influence of Torque Variation On Timing Belt Drive's Load Distribution,”. The 7th International Conference Research And Development of Mechanical Elements And Systems, 2011,pp.559-562.

[7] Jiazhen Hong, "Computational Dynamics of Multibody Systems," Beijing: Higher Education Press, 1999.

[8] Tingqiang Yao, Yilin Chi, Yayu Huang, et al. "Research on multi body dynamics and contact vibration of belt drive system," Journal of system simulation, vol.21,No.16,Aug.2009, pp.4945-4950,doi: 1004-731X (2009) 16-4945-06.

[9] Jiazhen Hong, Qing Yu. "Recursive modeling and algorithm of the flexible multi-body system dynamics," China mechanical engineering, vol.11, No.6, Jun.2000, pp.611-615, doi:1004-132X (2000) 06-0611-05.

[10] Yuechen Duan, Dingguo Zhang, Jiazhen Hong. "Partition method for impact dynamics of flexible multibody systems based on contact constraint," Department of Engineering Mechanics, vol.34, No.11, 2013,pp.1393-1404, doi: 10.1007/s10483-013-1754-7. 